\title{
Stability analysis of fractional switched systems with stable and unstable subsystems
}

\author{
Ran Yang, Song Liu, Xiaoyan Li, Jian Xiao \\ School of Mathematical Sciences, Anhui University, Hefei 230601, PR China
}

\begin{abstract}
This article addresses stability of fractional switched systems (FSSs) with stable and unstable subsystems. First, several algebraic conditions are presented to guarantee asymptotic stability by applying multiple Lyapunov function (MLF) method, dwell time technique and fast-slow switching mechanism. Then, some stability conditions which have less conservation are also provided by utilizing average dwell time (ADT) technique and the property of Mittag-Leffler function. In addition, sufficient conditions on asymptotic stability of delayed FSSs are obtained by virtue of fractional Razumikhin technique. Finally, several examples are given to reveal that the conclusions obtained are valid.
\end{abstract}

Keywords: Asymptotic stability; Fractional switched system; Multiple Lyapunov function method; Dell time; Fractional Razumikhin technique

\section{Introduction}

In recent years, many efforts focus on analysis and applications of switched systems because they can be used in various fields including electric cars [1], electric aircraft [2], genetic regulatory networks [3] and so on. In general, switched systems consists of a switching rule and a number of subsystems, which have some interesting and complicated dynamical behaviors, a switched system may be unstable under some switching rule even if its all subsystems are stable [4]. Most existing results pay attention to stability of switched systems which have only stable subsystems, common Lyapunov function(CLF) approach [5] and $\operatorname{MLF}$ approach $[6,7,8]$ are main methods. In many actual circumstances, however, both stable and unstable subsystems may be contained in a switched system, the fast-slow switching scheme is often used to deal with stability of such systems. Based on fast-slow switching scheme and MLF and mode-dependent ADT approach, stability of linear switched systems [9], singular switched systems [10], linear and nonlinear delayed switched systems $[11,12]$ is solved.

The above papers only concern integer-order dynamical models, however, fractional calculus

\footnotetext{
${ }^{*}$ Corresponding author. E-mail address: liusong@ahu.edu.cn (S. Liu).
} 
has shown outstanding advantages on depicting many complicated process and phenomena [13, 14] during past thirty years. The complexity of fractional calculus and switching rules bring great difficulties on the stability analysis, many efforts are paid to deal with stability of FSSs with stable subsystems. The stability under arbitrary switching is investigated in by employing CLF [15, 16] and MLF [17] method. Some other approaches including state-dependent switching [18], ADT $[19,20,21]$ and mode-dependent ADT [22, 23] are also adopted. In [24], the formulas of solutions are provided to deal with finite-time stability of FSSs, $[25,26]$ investigate stability of impulsive FSSs including unstable and stable subsystems by utilizing MLF approach and dwell time technique.

It is noteworthy that time delays are usually inevitable for many actual systems, which usually have particular impact on sytems' dynamics, Nguyen et al. [27] design a state-dependent switching law to implement finite-time stability of delayed singular FSSs. Employing integer-order Lyapunov function method, an observer-based controller is chosen to analyze stability of Riemann-Liouville delayed FSSs [28]. He et al. [29] provide fractional differential inequalities to study exponential stability of impulsive delayed FSSs. It should be noted that time delays usually take on a spatial extent and the real systems cannot be well modeled by dynamical models with discrete delays, but distributed delays may better describe such complicated lag phenomena and have some important applications [30,31]. Up to now, there are no effective time-domain ways to handle stability of FSSs with distributed delay in Caputo sense.

Inspired by the above analysis, asymptotic stability of FSSs with stable and unstable subsystems is first investigated in this paper. Sufficient conditions are presented by utilizing MLF method, dwell time technique and fast-slow switching mechanism. Then, a less conservative criterion is provided by employing ADT technique and the property of Mittag-Leffler functions. Finally, a class of delayed FSSs are addressed and algebraic conditions on asymptotic stability are given. The highlights of this paper are listed as follows: 1) Two different approaches are developed to deal with stability of FSSs and several simple algebraic criteria are provided. 2) FSSs with distributed and discrete delays are addressed, an effective approach is developed to overcome the difficulties arising from time delays, fractional calculus and switching rules with the help of fractional Razumikhin technique, MLF method and dwell time.

\section{Preparatory knowledge}

\subsection{Preliminaries}

In this subsection, several necessary definitions and useful lemmas are first stated.

Definition 1 [32]. The $\mu$-order $(0<\mu<1)$ Caputo derivative of $\varphi(t)$ is given by

$$
D^{\mu} \varphi(t)=\frac{1}{\Gamma(1-\mu)} \int_{t_{0}}^{t} \frac{\varphi^{\prime}(s)}{(t-s)^{\mu}} d s .
$$


Definition 2 [32]. The $\mu$-order Riemann-Liouville integral of $\varphi(t)$ is given by

$$
I^{\mu} \varphi(t)=\frac{1}{\Gamma(\mu)} \int_{t_{0}}^{t}(t-s)^{\mu-1} \varphi(s) d s, \quad \mu>0 .
$$

Definition 3 [32]. A Mittag-Leffler function with two parameters is given by

$$
E_{\alpha, \beta}(y)=\sum_{j=0}^{\infty} \frac{y^{j}}{\Gamma(\alpha j+\beta)}, \quad \alpha, \beta>0 .
$$

Lemma 1 [19]. From the Definitions 1 and 2 one has for $0<\mu<1$,

$$
I^{\mu}\left(D^{\mu} \varphi(t)\right)=\varphi(t)-\varphi\left(t_{0}\right), \quad t \geq t_{0} .
$$

Lemma 2 [33]. For an absolutely continuous function $\varphi(t) \in \mathbf{R}^{n}$, one has

$$
D^{\mu}\left(\varphi^{T}(t) \varphi(t)\right) \leq 2 \varphi^{T}(t) D^{\mu} \varphi(t), \quad 0<\mu<1
$$

Lemma 3 [34]. Let $\alpha_{1}, \alpha_{2} \in \mathbf{R}^{n}$ and $\kappa>0$, the following inequality holds

$$
2 \alpha_{1}^{\mathrm{T}} \alpha_{2} \leq \kappa \alpha_{1}^{\mathrm{T}} \alpha_{1}+\frac{1}{\kappa} \alpha_{2}^{\mathrm{T}} \alpha_{2}
$$

Lemma 4 [35]. Let $\omega(t)$ satisfy $\omega(t) \leq \int_{0}^{t} b(\zeta) \omega(\zeta) d \zeta+c(t)$, where $b(t)$ and $c(t)$ are known real functions, then

$$
\omega(t) \leq \int_{0}^{t} b(\zeta) c(\zeta) \exp \left\{\int_{\zeta}^{t} b(s) d s\right\} d \zeta+c(t) .
$$

In particular, if $c(t)=c$ is a constant, one has

$$
\omega(t) \leq c \cdot \exp \left\{\int_{0}^{t} b(\zeta) d \zeta\right\}
$$

Lemma 5 [36]. For any matrix $A_{0}$ and constant $\mu \in(0,1)$, there is a constant $k \geq 1$ satisfying

$$
\left\|E_{\mu, 1}\left(A_{0} t^{\mu}\right)\right\| \leq k\left\|\exp \left\{A_{0} t\right\}\right\|
$$

\subsection{Problem Statement}

Consider the following FSS composed of $M$ subsystems:

$$
D^{\mu} y(t)=A_{\eta(t)} y(t)+f_{\eta(t)}(t, y(t)), \quad t \geq t_{0},
$$

where $y(t) \in \mathbf{R}^{n}, A_{\eta(t)} \in \mathbf{R}^{n \times n}$ are constant matrices. $\eta:\left[t_{0}, \infty\right) \rightarrow \mathcal{F}=\{1,2, \cdots, M\}$ is a piecewise switching signal, $\eta(t)=i \in \mathcal{F}$ for $t \in\left[t_{q}, t_{q+1}\right), q=0,1,2, \cdots$ means that the $i$ th 
subsystem is active, where $t_{q}, q \in \mathbf{Z}^{+} \cup\{0\}$ is the switching instant and $\lim _{q \rightarrow+\infty} t_{q}=+\infty$. We suppose that Eq.(10) has $L(1 \leq L \leq M)$ stable subsystems and $M-L$ unstable subsystems, $S:=\{1,2, \cdots, L\}$ and $U:=\{L, L+1, \cdots, M\}$ denote the set of stable and unstable subsystems, $S \cup L=\mathcal{F}$. For all $i \in \mathcal{F}, f_{i}: \mathbf{R} \times \mathbf{R}^{n} \rightarrow \mathbf{R}^{n}$ satisfy $f_{i}(t, 0)=0$ and

$$
\left\|f_{i}\left(t, \omega_{1}\right)-f_{i}\left(t, \omega_{2}\right)\right\| \leq h_{i}\left\|\omega_{1}-\omega_{2}\right\|
$$

where $h_{i}>0$.

Remark 1. Here we take $y\left(t_{0}\right)$ as the initial state of the first activated subsystem and the terminal state $y\left(t_{q}^{-}\right)$on interval $\left[t_{q-1}, t_{q}\right)$ as the initial state on next interval $\left[t_{q}, t_{q+1}\right)$ (see also $[20,21,25,26]$ ).

\section{Main results}

\subsection{Asymptotical stability of the FSS with unstable subsystems}

This subsection will give several algebraic conditions and lower and upper bounds of dwell times to ensure asymptotic stability of FSS (10) by employing MLF approach, dwell time technique and fast-slow switching mechanism. Let positive scalars $\tau_{s}$ and $\tau_{u}$ be the dwell times of stable and unstable subsystems, respectively. Here $t_{q+1}-t_{q} \geq \tau_{s}$ and $t_{q+1}-t_{q} \leq \tau_{u}$ for any two consecutive switchings $t_{q}, t_{q+1}$, we now may provide our main results.

Theorem 1. FSS (10) will be asymptotically stable if there are scalars $\xi_{s}, \xi_{u}>0, \nu_{s}>1,0<\nu_{u}<1$ and positive definite matrices $P_{i}, P_{j}$ such that

$$
\begin{gathered}
\Delta_{i}^{1}+\xi_{s} P_{i} \leq 0, \quad i \in S, \\
\Delta_{i}^{1}-\xi_{u} P_{i} \leq 0, \quad i \in U, \\
P_{i} \leq \nu_{s} P_{j} \text { for any } i \in S, j \in \mathcal{F} \text { and } P_{i} \leq \nu_{u} P_{j} \text { for any } i \in U, j \in S,
\end{gathered}
$$

where $\Delta_{i}^{1}=P_{i} A_{i}+A_{i}^{\mathrm{T}} P_{i}+P_{i}^{2}+h_{i}^{2} I_{n}$, and the dwell times satisfy

$$
\begin{gathered}
\tau_{s}>\left(\frac{\ln \nu_{s} \Gamma(\mu+1)}{\xi_{s}}\right)^{\frac{1}{\mu}}, \\
\tau_{u}<\left(\frac{\ln \frac{1}{\nu_{u}} \Gamma(\mu+1)}{\xi_{u}}\right)^{\frac{1}{\mu}} .
\end{gathered}
$$

Proof. Assuming $\eta(t)=i \in \mathcal{F}$ for $t \in\left[t_{q}, t_{q+1}\right)$, writing $y(t)$ as $y$. Then constructing the MLF

$$
V_{i}(y)=y^{\mathrm{T}} P_{i} y .
$$


From Lemma 2, one has

$$
\begin{aligned}
D^{\mu} V_{i}(y) & =D^{\mu}\left(y^{\mathrm{T}} P_{i} y\right) \leq 2 y^{\mathrm{T}} P_{i} D^{\mu} y \\
& =2 y^{\mathrm{T}} P_{i}\left[A_{i} y+f_{i}(t, y)\right] \\
& =y^{\mathrm{T}}\left[P_{i} A_{i}+A_{i}^{\mathrm{T}} P_{i}\right] y+2 y^{\mathrm{T}} P_{i} f_{i}(t, y) .
\end{aligned}
$$

Based on Lemma 3 and relationship (11), we can obtain that

$$
\begin{aligned}
D^{\mu} V_{i}(y) & \leq y^{\mathrm{T}}\left[P_{i} A_{i}+A_{i}^{\mathrm{T}} P_{i}\right] y+y^{\mathrm{T}} P_{i}^{2} y+f_{i}^{\mathrm{T}}(t, y) f_{i}(t, y) \\
& \leq y^{\mathrm{T}}\left[P_{i} A_{i}+A_{i}^{\mathrm{T}} P_{i}+P_{i}^{2}\right] y+h_{i}^{2} y^{\mathrm{T}} y \\
& =y^{\mathrm{T}}\left[P_{i} A_{i}+A_{i}^{\mathrm{T}} P_{i}+P_{i}^{2}+h_{i}^{2} I_{n}\right] y
\end{aligned}
$$

Defining $\xi_{i}=-\xi_{s}, i \in S$ and $\xi_{i}=\xi_{u}, i \in U$, we get by the inequalities (12) and (13) that

$$
D^{\mu} V_{i}(y) \leq \xi_{i} V_{i}(y)=\left\{\begin{array}{c}
-\xi_{s} V_{i}(y), i \in S \\
\xi_{u} V_{i}(y), i \in U .
\end{array}\right.
$$

From Lemma 1, taking the $\mu$-order integral on both sides of inequality (20) from $t_{q}$ to $t$ for any $t \in\left[t_{q}, t_{q+1}\right)$ yields

$$
V_{i}(y) \leq V_{i}\left(y\left(t_{q}\right)\right)+\frac{1}{\Gamma(\mu)} \int_{t_{q}}^{t} \frac{\xi_{i} V_{i}(y(s))}{(t-s)^{1-\mu}} d s .
$$

In the light of Lemma 4, one has

$$
\begin{aligned}
V_{i}(y) & \leq V_{i}\left(y\left(t_{q}\right)\right) \exp \left\{\frac{\xi_{i}}{\Gamma(\mu)} \int_{t_{q}}^{t}(t-s)^{\mu-1} d s\right\} \\
& =V_{i}\left(y\left(t_{q}\right)\right) \exp \left\{\frac{\xi_{i}}{\Gamma(\mu+1)}\left(t-t_{q}\right)^{\mu}\right\} .
\end{aligned}
$$

Without loss of generality, we suppose that $l$ stable and $(q-l)$ unstable subsystems have been activated before switching instant $t_{q}$, then one has from (14) and (22)

$$
\begin{aligned}
V_{\eta\left(t_{q}\right)}\left(y\left(t_{q}\right)\right) & \leq \nu_{\eta\left(t_{q}\right)} V_{\eta\left(t_{q-1}\right)}\left(y\left(t_{q}^{-}\right)\right) \\
& \leq \nu_{\eta\left(t_{q}\right)} V_{\eta\left(t_{q-1}\right)}\left(y\left(t_{q-1}\right)\right) \exp \left\{\frac{\xi_{\eta\left(t_{q-1}\right)}}{\Gamma(\mu+1)}\left(t_{q}-t_{q-1}\right)^{\mu}\right\} \\
& \leq \nu_{\eta\left(t_{q}\right)} \nu_{\eta\left(t_{q-1}\right)} V_{\eta\left(t_{q-1}^{-}\right)}\left(y\left(t_{q-1}^{-}\right)\right) \exp \left\{\frac{\xi_{\eta\left(t_{q-1}\right)}}{\Gamma(\mu+1)}\left(t_{q}-t_{q-1}\right)^{\mu}\right\}
\end{aligned}
$$




$$
\begin{aligned}
& \leq \nu_{\eta\left(t_{q}\right)} \nu_{\eta\left(t_{q-1}\right)} V_{\eta\left(t_{q-2}\right)}\left(y\left(t_{q-2}\right)\right) \exp \left\{\frac{\xi_{\eta\left(t_{q-1}\right)}}{\Gamma(\mu+1)}\left(t_{q}-t_{q-1}\right)^{\mu}+\frac{\xi_{\eta\left(t_{q-2}\right)}}{\Gamma(\mu+1)}\left(t_{q-1}-t_{q-2}\right)^{\mu}\right\} \\
& \leq \cdots \\
& \leq \Pi_{i=1}^{q} \nu_{\eta\left(t_{i}\right)} V_{\eta\left(t_{0}\right)}\left(y\left(t_{0}\right)\right) \exp \left\{\frac{\xi_{\eta\left(t_{q-1}\right)}}{\Gamma(\mu+1)}\left(t_{q}-t_{q-1}\right)^{\mu}+\cdots+\frac{\xi_{\eta\left(t_{0}\right)}}{\Gamma(\mu+1)}\left(t_{1}-t_{0}\right)^{\mu}\right\} \\
& =\nu_{s}^{l} \nu_{u}^{q-l} V_{\eta\left(t_{0}\right)}\left(y\left(t_{0}\right)\right) \exp \left\{\frac{\xi_{\eta\left(t_{q-1}\right)}}{\Gamma(\mu+1)}\left(t_{q}-t_{q-1}\right)^{\mu}+\cdots+\frac{\xi_{\eta\left(t_{0}\right)}}{\Gamma(\mu+1)}\left(t_{1}-t_{0}\right)^{\mu}\right\}
\end{aligned}
$$

Noticing that $t_{q}-t_{q-1} \geq \tau_{s}$ and $t_{q}-t_{q-1} \leq \tau_{u}$, one has

$$
\exp \left\{\frac{\xi_{\eta\left(t_{q-1}\right)}}{\Gamma(\mu+1)}\left(t_{q}-t_{q-1}\right)^{\mu}\right\} \leq\left\{\begin{array}{l}
\exp \left\{-\frac{\xi_{s}}{\Gamma(\mu+1)} \tau_{s}^{\mu}\right\}, \text { if } \eta(t) \in S \\
\exp \left\{\frac{\xi_{u}}{\Gamma(\mu+1)} \tau_{u}^{\mu}\right\}, \text { if } \eta(t) \in U
\end{array}\right.
$$

It follows that

$$
V_{\eta\left(t_{q}\right)}\left(y\left(t_{q}\right)\right) \leq V_{\eta\left(t_{0}\right)}\left(y\left(t_{0}\right)\right)\left[\nu_{s} \exp \left\{-\frac{\xi_{s}}{\Gamma(\mu+1)} \tau_{s}^{\mu}\right\}\right]^{l}\left[\nu_{u} \exp \left\{\frac{\xi_{u}}{\Gamma(\mu+1)} \tau_{u}^{\mu}\right\}\right]^{q-l} .
$$

Inequalities (15) and (16) mean $\nu_{s} \exp \left\{-\frac{\xi_{s}}{\Gamma(\mu+1)} \tau_{s}^{\mu}\right\}<1$ and $\nu_{u} \exp \left\{\frac{\xi_{u}}{\Gamma(\mu+1)} \tau_{u}^{\mu}\right\}<1$, therefore, we can get $\lim _{t \rightarrow+\infty} V_{\eta(t)}(y)=0$ from $\lim _{q \rightarrow+\infty} V_{\eta\left(t_{q}\right)}\left(y\left(t_{q}\right)\right)=0$, which yields $y \rightarrow 0$ as $t \rightarrow+\infty$. Finally, we conclude that FSS (10) is asymptotically stable.

Remark 2. The inequality $P_{i} \leq \nu_{s} P_{j}, \nu_{s}>1$ of relationship (14) implies that it is impossible that MLF (17) always decreases on the switching sequence $\left\{t_{q}, q=0,1, \cdots\right\}$ because the FSS has only finite different subsystems, the MLF must increase at some switching instants. Otherwise, if the $m$ th and $l$ th subsystems are sequentially activated at $t_{q-1}, t_{q}, t_{q+1}$, then, there is a scalar $0<\nu_{u}<1$ satisfying $V_{l}\left(y\left(t_{q}\right)\right) \leq \nu_{u} V_{m}\left(y\left(t_{q}^{-}\right)\right)$and $V_{m}\left(y\left(t_{q+1}\right)\right) \leq \nu_{u} V_{l}\left(y\left(t_{q+1}^{-}\right)\right)$, which means $P_{l} \leq \nu_{u} P_{m}$ and $P_{m} \leq \nu_{u} P_{l}$, this concludes a contradictory.

On the other hand, to get a upper bound of $\tau_{u}$, we need that the MLF decrease at switching instants when unstable subsystems are activated. However, unstable subsystems cannot be consecutively activated according to above analysis, hence the relationship $P_{i} \leq \nu_{u} P_{j}$ for any $i \in U, j \in S$ is necessary. It follows that the MLF will increase on some switching instants and time intervals. To make the MLF converge to 0, we need eliminate these increments, this may be completed by the decrements brought by stable subsystems. Therefore, stable subsystems must operate long time enough and unstable subsystems stay a shorter time and hereby we provide the lower bound of $\tau_{s}$ and upper bound of $\tau_{u}$.

In order to derive a less conservative criterion, we introduce the following definition.

Definition 4. For any $t_{2} \geq t_{1} \geq 0$, denote $N_{u}\left(t_{1}, t_{2}\right)$ or $N_{s}\left(t_{1}, t_{2}\right)$ as the switching numbers of active unstable or stable subsystems in interval $\left[t_{1}, t_{2}\right]$, and $T_{u}\left(t_{1}, t_{2}\right)$ or $T_{s}\left(t_{1}, t_{2}\right)$ as the total running time 
of active unstable or stable subsystems. If there exist $N_{0 p} \geq 0$ and $\tau_{d p}>0, p=\{s, u\}$ such that

$$
N_{p}\left(t_{1}, t_{2}\right) \leq N_{0 p}+\frac{T_{p}\left(t_{1}, t_{2}\right)}{\tau_{d p}},
$$

then $\tau_{d s}$ and $\tau_{d u}$ are called the ADTs of stable and unstable subsystems, respectively, $N_{0 p}$ are called the chatter bounds, here we choose $N_{0 p}=0$.

Theorem 2. FSS (10) will be asymptotically stable if there are scalars $\xi_{s}, \xi_{u}>0, \nu>1, k \geq 1$ and positive definite matrices $P_{i}, P_{j}$ such that

$$
\begin{gathered}
\Delta_{i}^{1}+\xi_{s} P_{i} \leq 0, \quad i \in S, \\
\Delta_{i}^{1}-\xi_{u} P_{i} \leq 0, \quad i \in U, \\
P_{i} \leq \nu P_{j}, \quad i, j \in \mathcal{F},
\end{gathered}
$$

and

$$
\tau_{d s}>\frac{\ln k \nu}{\xi_{s}}, \quad \frac{T_{u}}{T_{s}} \leq \frac{\alpha_{s}-\alpha}{\alpha_{u}+\alpha}, 0<\alpha<\alpha_{s}
$$

where $\Delta_{i}^{1}=P_{i} A_{i}+A_{i}^{\mathrm{T}} P_{i}+P_{i}^{2}+h_{i}^{2} I_{n}, \alpha_{s}=\xi_{s}-\frac{\ln k \nu}{\tau_{d s}}$ and $\alpha_{u}=\xi_{u}+\frac{\ln k \nu}{\tau_{d u}}, T_{u}$ or $T_{s}$ is total running time of unstable or stable subsystems.

Proof. Similarly to Theorem 1, according to (27) and (28), the $\mu$-order derivative of MLF (17) is given by

$$
D^{\mu} V_{i}(y) \leq \xi_{i} V_{i}(y)=\left\{\begin{array}{c}
-\xi_{s} V_{i}(y), i \in S \\
\xi_{u} V_{i}(y), i \in U
\end{array}\right.
$$

where $\xi_{i}=-\xi_{s}$ for $i \in S$ and $\xi_{i}=\xi_{u}$ for $i \in U$.

Hence, there is a nonnegative function $W(t)$ satisfying

$$
D^{\mu} V_{i}(y)+W(t)=\xi_{i} V_{i}(y), \quad t \in\left[t_{q}, t_{q+1}\right)
$$

According to the equation (4.1.62) of [37], the solution to (32) is expressed by

$$
\left.V_{i}(y)=V_{i}\left(y\left(t_{q}\right)\right) E_{\mu, 1}\left(\xi_{i}\left(t-t_{q}\right)^{\mu}\right)-\int_{t_{q}}^{t}(t-s)^{\mu-1} E_{\mu, \mu}\left(\xi_{i}(t-s)^{\mu}\right)\right) W(s) d s .
$$

Because $\left(t-t_{q}\right)^{\mu-1}$ and $E_{\mu, \mu}\left(\xi_{i}\left(t-t_{q}\right)^{\mu}\right)$ are nonnegative functions, one has

$$
V_{i}(y) \leq V_{i}\left(y\left(t_{q}\right)\right) E_{\mu, 1}\left(\xi_{i}\left(t-t_{q}\right)^{\mu}\right)
$$


Noticing $\eta(t)=i, t \in\left[t_{q}, t_{q+1}\right)$, hence for any $t \in\left[t_{q}, t_{q+1}\right)$, we have from (29) and (34)

$$
\begin{aligned}
& V_{\eta\left(t_{q}\right)}(y) \\
& \leq V_{\eta\left(t_{q}\right)}\left(y\left(t_{q}\right)\right) E_{\mu, 1}\left(\xi_{\eta\left(t_{q}\right)}\left(t-t_{q}\right)^{\mu}\right) \\
& \leq \nu V_{\eta\left(t_{q-1}\right)}\left(y\left(t_{q}^{-}\right)\right) E_{\mu, 1}\left(\xi_{\eta\left(t_{q}\right)}\left(t-t_{q}\right)^{\mu}\right) \\
& \leq \nu V_{\eta\left(t_{q-1}\right)}\left(y\left(t_{q-1}\right)\right) E_{\mu, 1}\left(\xi_{\eta\left(t_{q}-1\right)}\left(t_{q}-t_{q-1}\right)^{\mu}\right) E_{\mu, 1}\left(\xi_{\eta\left(t_{q}\right)}\left(t-t_{q}\right)^{\mu}\right) \\
& \leq \nu^{2} V_{\eta\left(t_{q-2}\right)}\left(y\left(t_{q-1}^{-}\right)\right) E_{\mu, 1}\left(\xi_{\eta\left(t_{q}-1\right)}\left(t_{q}-t_{q-1}\right)^{\mu}\right) E_{\mu, 1}\left(\xi_{\eta\left(t_{q}\right)}\left(t-t_{q}\right)^{\mu}\right) \\
& \leq \nu^{2} V_{\eta\left(t_{q-2}\right)}\left(y\left(t_{q-2}\right)\right) E_{\mu, 1}\left(\xi_{\eta\left(t_{q}-2\right)}\left(t_{q-1}-t_{q-2}\right)^{\mu}\right) E_{\mu, 1}\left(\xi_{\eta\left(t_{q}-1\right)}\left(t_{q}-t_{q-1}\right)^{\mu}\right) E_{\mu, 1}\left(\xi_{\eta\left(t_{q}\right)}\left(t-t_{q}\right)^{\mu}\right) \\
& \leq \cdots \\
& \leq \nu^{N_{s}\left(t_{0}, t\right)+N_{u}\left(t_{0}, t\right)} V_{\eta\left(t_{0}\right)}\left(y\left(t_{0}\right)\right) E_{\mu, 1}\left(\xi_{\eta\left(t_{0}\right)}\left(t_{1}-t_{0}\right)^{\mu}\right) \cdots E_{\mu, 1}\left(\xi_{\eta\left(t_{q}\right)}\left(t-t_{q}\right)^{\mu}\right) .
\end{aligned}
$$

It follows that from Lemma 5 and relationship (26)

$$
\begin{aligned}
\left\|V_{\eta\left(t_{q}\right)}(y)\right\| & \leq\left\|\nu^{N_{s}\left(t_{0}, t\right)+N_{u}\left(t_{0}, t\right)} V_{\eta\left(t_{0}\right)}\left(y\left(t_{0}\right)\right)\right\|\left\|E_{\mu, 1}\left(\xi_{\eta\left(t_{0}\right)}\left(t_{1}-t_{0}\right)^{\mu}\right)\right\| \cdots\left\|E_{\mu, 1}\left(\xi_{\eta\left(t_{q}\right)}\left(t-t_{q}\right)^{\mu}\right)\right\| \\
& \leq \nu^{\frac{T_{s}}{\tau_{d s}}+\frac{T_{u}}{\tau_{d u}}}\left\|V_{\eta\left(t_{0}\right)}\left(y\left(t_{0}\right)\right)\right\| \cdot k \exp \left\{\xi_{\eta\left(t_{0}\right)}\left(t_{1}-t_{0}\right)\right\} \cdots k \exp \left\{\xi_{\eta\left(t_{q}\right)}\left(t-t_{q}\right)\right\} \\
& =\nu^{\frac{T_{s}}{\tau_{d s}}+\frac{T_{u}}{\tau_{d u}} \cdot k^{\frac{T_{s}}{\tau_{d s}}}+\frac{T_{u}}{\tau_{d u}}+1}\left\|V_{\eta\left(t_{0}\right)}\left(y\left(t_{0}\right)\right)\right\| \exp \left\{-\xi_{s} T_{s}+\xi_{u} T_{u}\right\} \\
& =k(k \nu)^{\frac{T_{s}}{\tau_{d s}}}+\frac{T_{u}}{\tau_{d u}}\left\|V_{\eta\left(t_{0}\right)}\left(y\left(t_{0}\right)\right)\right\| \exp \left\{-\xi_{s} T_{s}+\xi_{u} T_{u}\right\} \\
& =k\left\|V_{\eta\left(t_{0}\right)}\left(y\left(t_{0}\right)\right)\right\| \exp \left\{\left(\frac{T_{s}}{\tau_{d s}}+\frac{T_{u}}{\tau_{d u}}\right) \ln k \nu\right\} \exp \left\{-\xi_{s} T_{s}+\xi_{u} T_{u}\right\} \\
& =k\left\|V_{\eta\left(t_{0}\right)}\left(y\left(t_{0}\right)\right)\right\| \exp \left\{\left(-\xi_{s}+\frac{\ln k \nu}{\tau_{d s}}\right) T_{s}+\left(\xi_{u}+\frac{\ln k \nu}{\tau_{d u}}\right) T_{u}\right\} \\
& =k\left\|V_{\eta\left(t_{0}\right)}\left(y\left(t_{0}\right)\right)\right\| \exp \left\{-\alpha_{s} T_{s}+\alpha_{u} T_{u}\right\} .
\end{aligned}
$$

Inequality (30) means that there is $0<\alpha<\alpha_{s}$ such that

$$
\exp \left\{-\alpha_{s} T_{s}+\alpha_{u} T_{u}\right\} \leq \exp \left\{-\alpha\left(T_{s}+T_{u}\right)\right\}=\exp \left\{-\alpha\left(t-t_{0}\right)\right\}
$$

Submitting (37) into (36) yields

$$
\left\|V_{\eta\left(t_{q}\right)}(y)\right\| \leq k\left\|V_{\eta\left(t_{0}\right)}\left(y\left(t_{0}\right)\right)\right\| \exp \left\{-\alpha\left(t-t_{0}\right)\right\}
$$

It is obvious that $\lim _{t \rightarrow+\infty} V_{\eta(t)}(y)=0$, which yields $\lim _{t \rightarrow+\infty} y=0$, therefore FSS (10) is asymptotically stable.

Remark 3. Be different from Theorem 1, the unstable subsystems can be consecutively activated in Theorem 2 since inequality (29) implies that the MLF will increase at these switching instants.

Remark 4. In [25], the authors obtain a more complex condition on dwell time to achieve the asymptotic stability of FSSs with unstable subsystems, which is described as an inequality of 
multiplication of $N$ Mittag-Leffler functions. [26] considers stability of impulsive FSSs with unstable subsystems, however, all impulsive strengths must be less than zero, which has great conservation and the method is not suitable for FSSs without impulses. Here, two simple upper and lower bounds of dwell times are proposed to ensure the stability.

\subsection{Asymptotical stability of delayed FSSs with unstable subsystems}

Consider the following nonlinear delayed FSS for $i \in \mathcal{F}$ :

$$
\left\{\begin{array}{l}
D^{\mu} y=A_{i} y+\int_{-r_{i}}^{0} B_{i} y(t+\xi) d \xi+f_{i}\left(t, y, y\left(t-r_{i}\right)\right), \\
y\left(t_{0}+\theta\right)=\psi(\theta), \quad \forall \theta \in[-\bar{r}, 0]
\end{array}\right.
$$

where $y(\cdot) \in \mathbf{R}^{n}, A_{i}, B_{i} \in \mathbf{R}^{n \times n}$ are constant matrices, $r_{i}>0$ are constant delays, $\bar{r}=\max _{i \in \mathcal{F}}\left\{r_{i}\right\}$, $f_{i}: \mathbf{R} \times \mathbf{R}^{n} \times \mathbf{R}^{n} \rightarrow \mathbf{R}^{n}$ satisfy

$$
\left\|f_{i}\left(t, \omega_{1}, \omega_{1}\left(t-r_{i}\right)\right)-f_{i}\left(t, \omega_{2}, \omega_{2}\left(t-r_{i}\right)\right)\right\| \leq h_{i}\left\|\omega_{1}-\omega_{2}\right\|+l_{i}\left\|\omega_{1}\left(t-r_{i}\right)-\omega_{2}\left(t-r_{i}\right)\right\|,
$$

and $f_{i}(t, 0,0)=0$, where $h_{i}, l_{i}>0$.

To obtain our main results, a useful lemma will be introduced.

Denote $\mathcal{C}=C\left([-r, 0] \rightarrow \mathbf{R}^{n}\right)$, consider the $\mu$-order delayed system

$$
\left\{\begin{array}{l}
D^{\mu} y(t)=h\left(t, y_{t}\right), \quad t \geq t_{0}, \\
y_{t_{0}}(\theta)=\psi(\theta), \quad \theta \in[-r, 0],
\end{array}\right.
$$

where $0<\mu<1, r>0$ and $y_{t}(\theta)=y(t+\theta), \psi \in \mathcal{C}, \theta \in[-r, 0]$. The function $h: \mathbf{R} \times \mathcal{C} \rightarrow \mathbf{R}^{n}$ is Lipschitz continuous and $h(t, 0)=0$.

Lemma 6 [38]. The zero solution of system (41) will be stable if there are continuous nondecreasing functions $v_{1}, v_{2}, v_{3}: \mathbf{R}^{+} \rightarrow \mathbf{R}^{+}, v_{j}(s)>0$ for $s>0, j=1,2$ and $v_{1}(0)=v_{2}(0)=0$, and a consecutive function $V: \mathbf{R} \times \mathbf{R}^{n} \rightarrow \mathbf{R}$ satisfying for $y \in \mathbf{R}^{n}$

$$
v_{1}(\|y\|) \leq V(t, y) \leq v_{2}(\|y\|)
$$

whenever $V(t+\theta, y(t+\theta)) \leq V(t, y)$ for $\theta \in[-r, 0]$,

$$
\left.D^{\mu} V(t, y)\right|_{(41)} \leq-v_{3}(\|y\|)
$$

Theorem 3. FSS (39) will be asymptotically stable if there are scalars $\xi_{s}, \xi_{u}>0, \nu_{s}>1$, $0<\nu_{u}<1$ and positive definite matrices $P_{i}, P_{j}$ such that

$$
\Delta_{i}^{2}+\xi_{s} P_{i} \leq 0, \quad i \in S
$$




$$
\begin{gathered}
\Delta_{i}^{2}-\xi_{u} P_{i} \leq 0, \quad i \in U, \\
P_{i} \leq \nu_{s} P_{j} \text { for any } i \in S, j \in \mathcal{F} \text { and } P_{i} \leq \nu_{u} P_{j} \text { for any } i \in U, j \in S,
\end{gathered}
$$

where $\Delta_{i}^{2}=P_{i} A_{i}+A_{i}^{\mathrm{T}} P_{i}+\left(P_{i} B_{i} P_{i}^{-1} B_{i}^{\mathrm{T}} P_{i}+P_{i}\right) r_{i}+P_{i}^{2}+2 h_{i}^{2} I_{n}+\frac{2 l_{i}^{2}}{\lambda_{\min } P_{i}} P_{i}$, and the dwell times $\tau_{s}$ and $\tau_{u}$ of stable and unstable subsystems satisfy

$$
\begin{gathered}
\tau_{s}>\max \left\{\left(\frac{\ln \nu_{s} \Gamma(\mu+1)}{\xi_{s}}\right)^{\frac{1}{\mu}}, \bar{r}\right\}, \\
\tau_{u}<\left(\frac{\ln \frac{1}{\nu_{u}} \Gamma(\mu+1)}{\xi_{u}}\right)^{\frac{1}{\mu}} .
\end{gathered}
$$

Proof. According to Lemma 2, the $\mu$-order derivative of MLF (17) along system (39) is presented as follows

$$
\begin{aligned}
D^{\mu} V_{i}(y) & =D^{\mu}\left(y^{\mathrm{T}} P_{i} y\right) \leq 2 y^{\mathrm{T}} P_{i} D^{\mu} y \\
& =2 y^{\mathrm{T}} P_{i}\left[A_{i} y+\int_{-r_{i}}^{0} B_{i} y(t+\xi) d \xi+f_{i}\left(t, y, y\left(t-r_{i}\right)\right)\right] \\
& =y^{\mathrm{T}}\left[P_{i} A_{i}+A_{i}^{\mathrm{T}} P_{i}\right] y+\int_{-r_{i}}^{0} 2 y^{\mathrm{T}} P_{i} B_{i} y(t+\xi) d \xi+2 y^{\mathrm{T}} P_{i} f_{i}\left(t, y, y\left(t-r_{i}\right)\right) .
\end{aligned}
$$

Whenever

$$
V_{i}(t+\theta, y(t+\theta)) \leq V_{i}(t, y)
$$

that is,

$$
\lambda_{\min }\left(P_{i}\right) y^{\mathrm{T}}(t+\theta) y(t+\theta) \leq y^{\mathrm{T}}(t+\theta) P_{i} y(t+\theta) \leq y^{\mathrm{T}} P_{i} y, \theta \in[-\bar{r}, 0] .
$$

We can obtain that from Lemma 3 and (40)

$$
\begin{aligned}
D^{\mu} V_{i}(y) \leq & y^{\mathrm{T}}\left[P_{i} A_{i}+A_{i}^{\mathrm{T}} P_{i}\right] y+\int_{-r_{i}}^{0} y^{\mathrm{T}}\left[P_{i} B_{i} P_{i}^{-1} B_{i}^{\mathrm{T}} P_{i}\right] y d \xi \\
& +\int_{-r_{i}}^{0} y^{\mathrm{T}}(t+\xi) P_{i} y(t+\xi) d \xi+y^{\mathrm{T}} P_{i}^{2} y+f_{i}^{\mathrm{T}}\left(t, y, y\left(t-r_{i}\right)\right) f_{i}\left(t, y, y\left(t-r_{i}\right)\right) \\
\leq & y^{\mathrm{T}}\left[P_{i} A_{i}+A_{i}^{\mathrm{T}} P_{i}+P_{i} B_{i} P_{i}^{-1} B_{i}^{\mathrm{T}} P_{i} r_{i}+P_{i}^{2}\right] y \\
& +\int_{-r_{i}}^{0} y^{\mathrm{T}}(t+\xi) P_{i} y(t+\xi) d \xi+2 h_{i}^{2} y^{\mathrm{T}} y+2 l_{i}^{2} y^{\mathrm{T}}\left(t-r_{i}\right) y\left(t-r_{i}\right) .
\end{aligned}
$$

Submitting (51) into (52) yields

$$
\begin{aligned}
D^{\mu} V_{i}(y) \leq & y^{\mathrm{T}}\left[P_{i} A_{i}+A_{i}^{\mathrm{T}} P_{i}+P_{i} B_{i} P_{i}^{-1} B_{i}^{\mathrm{T}} P_{i} r_{i}+P_{i}^{2}\right] y \\
& +\int_{-r_{i}}^{0} y^{\mathrm{T}} P_{i} y d \xi+2 h_{i}^{2} y^{\mathrm{T}} y+\frac{2 l_{i}^{2}}{\lambda_{\min }\left(P_{i}\right)} y^{\mathrm{T}} P_{i} y \\
\leq & y^{\mathrm{T}}\left[P_{i} A_{i}+A_{i}^{\mathrm{T}} P_{i}+\left(P_{i} B_{i} P_{i}^{-1} B_{i}^{\mathrm{T}} P_{i}+P_{i}\right) r_{i}+P_{i}^{2}+2 h_{i}^{2} I_{n}+\frac{2 l_{i}^{2}}{\lambda_{\min } P_{i}} P_{i}\right] y .
\end{aligned}
$$


We can get that $D^{\mu} V_{i}(y) \leq-\xi_{s} V_{i}(y) \leq-\xi_{s} \lambda_{\min }\left(P_{i}\right)\|y\|^{2}$ from $\Delta_{i}^{2}+\xi_{s} P_{i} \leq 0$ of (44), which shows the zero solution of the $i$ th subsystem is stable by using Lemma 6 and thus $i \in S$.

Defining

$$
\xi_{i}=\left\{\begin{aligned}
-\xi_{s}, & i \in S, \\
\xi_{u}, & i \in U .
\end{aligned}\right.
$$

It follows that from inequalities (44) and (45)

$$
D^{\mu} V_{i}(y) \leq \xi_{i} V_{i}(y)=\left\{\begin{aligned}
-\xi_{s} V_{i}(y), & i \in S, \\
\xi_{u} V_{i}(y), & i \in U .
\end{aligned}\right.
$$

The rest of the proof is similar to Theorem 1, so it's omitted here.

Remark 5. Here we suppose that time delays are less than the dwell time $\tau_{s}$ of stable subsystems (see relationship (47)). Otherwise, the states of unstable systems may diverge to the infinity over time, for example, if FSS (39) contains only two subsystems in Example 1, one is stable and another is unstable, let $\bar{r}=0.9$ and the other parameters take the same values (the dwell time $\tau_{u}=0.25$ and $\tau_{s}=0.76$ ), the state trajectories of FSS (39) and the switching signal are described as the following Fig.A and Fig.B:

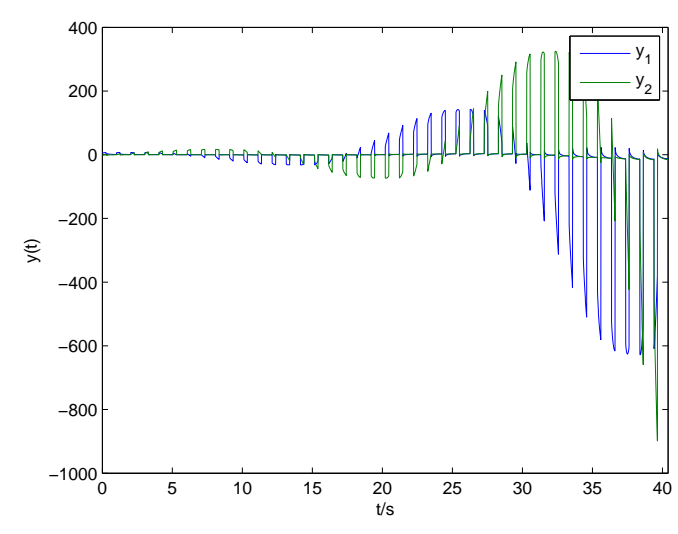

Fig.A

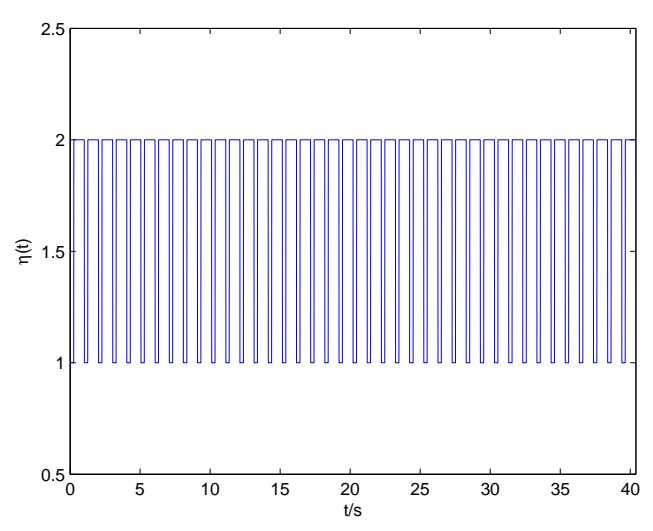

Fig.B

Theorem 4. FSS (39) will be asymptotically stable if there are scalars $\xi_{s}, \xi_{u}>0, \nu>1, k \geq 1$ and positive definite matrices $P_{i}, P_{j}$ such that

$$
\begin{gathered}
\Delta_{i}^{2}+\xi_{s} P_{i} \leq 0, \quad i \in S, \\
\Delta_{i}^{2}-\xi_{u} P_{i} \leq 0, \quad i \in U, \\
P_{i} \leq \nu P_{j}, \quad i, j \in \mathcal{F},
\end{gathered}
$$

and

$$
\tau_{d s}>\max \left\{\frac{\ln k \nu}{\xi_{s}}, \bar{r}\right\}, \frac{T_{u}}{T_{s}} \leq \frac{\alpha_{s}-\alpha}{\alpha_{u}+\alpha}, 0<\alpha<\alpha_{s} .
$$


where $\Delta_{i}^{2}=P_{i} A_{i}+A_{i}^{\mathrm{T}} P_{i}+\left(P_{i} B_{i} P_{i}^{-1} B_{i}^{\mathrm{T}} P_{i}+P_{i}\right) r_{i}+P_{i}^{2}+2 h_{i}^{2} I_{n}+\frac{2 l_{i}^{2}}{\lambda_{\min } P_{i}} P_{i}, \alpha_{s}=\xi_{s}-\frac{\ln k \nu}{\tau_{d s}}$ and $\alpha_{u}=\xi_{u}+\frac{\ln k \nu}{\tau_{d u}}, T_{u}$ or $T_{s}$ is total running time of unstable or stable subsystems.

Proof. The proof is similar to Theorem 3, so it is omitted here.

Remark 6. In [27], based on state-dependent switching, stability of FSSs with discrete delays is solved. [29] proposes a useful fractional differential inequality to analyze impulse FSSs with discrete delays. Riemann-Liouville delayed FSSs is studied in [28] by using integer-order Lyapunov function method, which can not be applicable to Caputo's sense systems since Caputo fractional operators have no composition property. Here, Caputo FSSs with discrete and distributed delays are addressed and its stability is solved by means of fractional Razumikhin technique and MLF method.

\section{Numerical Examples}

We now provide numerical examples to further demonstrate the availability of our main results. Example 1 (Unstable subsystems cannot be consecutively activated). Consider delayed FSS (39) equipped with two subsystems

$$
\begin{array}{rlr}
A_{1}=\left(\begin{array}{cc}
0.6 & -1.4 \\
1.5 & 0.8
\end{array}\right), & B_{1}=\left(\begin{array}{cc}
0.2 & 0.1 \\
0.15 & 0.25
\end{array}\right) . \\
A_{2}=\left(\begin{array}{cc}
-3.5 & 0.8 \\
0.4 & -2.5
\end{array}\right), & B_{2}=\left(\begin{array}{cc}
0.3 & 0.01 \\
0.2 & 0.1
\end{array}\right) .
\end{array}
$$

and $\bar{r}=0.5, f_{1}\left(t, y, y\left(t-r_{1}\right)\right)=\frac{1}{10} \sin y+\frac{2}{5} \tanh y\left(t-r_{1}\right), f_{2}\left(t, y, y\left(t-r_{2}\right)\right)=\frac{1}{5} \tanh y+\frac{1}{4} \sin y\left(t-r_{2}\right)$, thus $h_{1}=0.1, l_{1}=0.4, h_{2}=0.2$, and $l_{2}=0.25$ are chosen to meet (40).

Take $P_{1}=\left(\begin{array}{cc}0.4 & 0 \\ 0 & 0.35\end{array}\right)$ and $P_{2}=\left(\begin{array}{cc}1.6 & 0 \\ 0 & 1.5\end{array}\right)$, then $\nu_{u}=0.25$ and $\nu_{s}=4.3$ are picked to make $P_{1} \leq \nu_{u} P_{2}$ and $P_{2} \leq \nu_{s} P_{1}$, which means relationship (46) holds. Then we may take $\xi_{u}=3.78$ and $\xi_{s}=1.7$ to meet (44) and (45). 

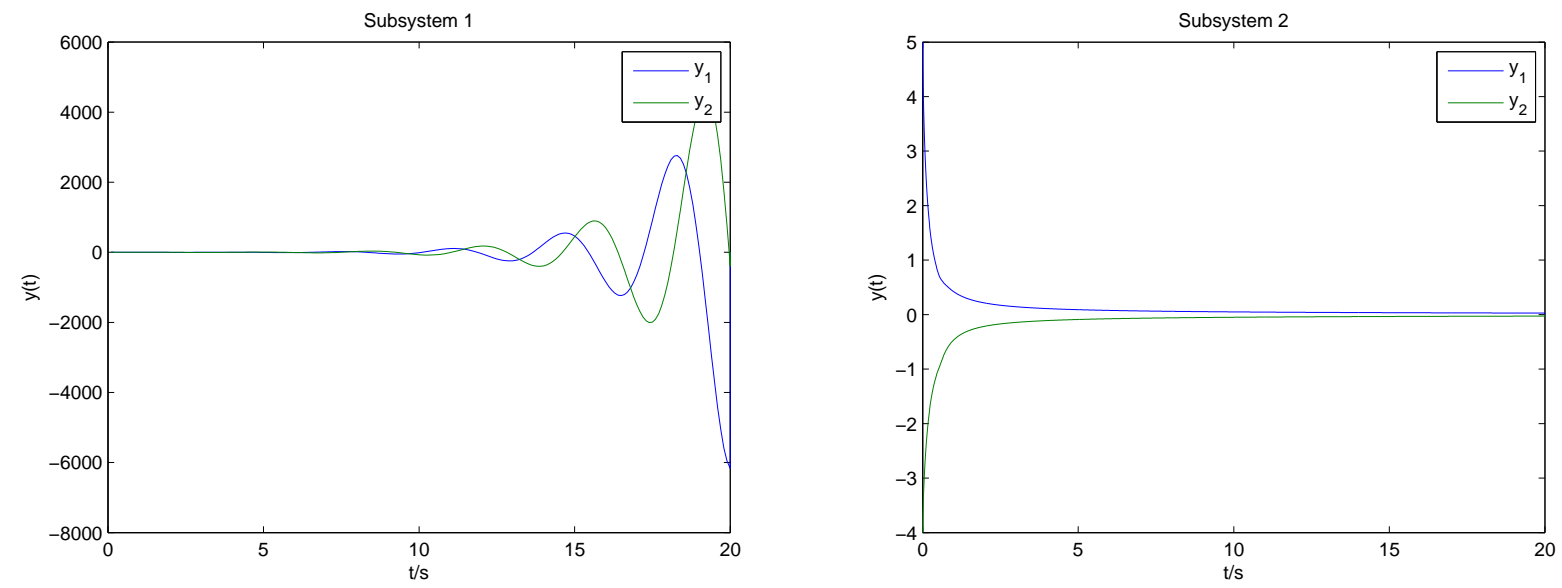

Fig.1. The state trajectories of two subsystems for delayed FSS (39).

Let $\mu=0.8$, through a series of simple calculations, the dwell time $\tau_{u}=0.25$ and $\tau_{s}=0.76$ can be taken in the light of $\tau_{u}<\left(\frac{\ln \frac{1}{\nu_{u}} \Gamma(\mu+1)}{\xi_{u}}\right)^{\frac{1}{\mu}}=0.2611$ and $\tau_{s}>\max \left\{\left(\frac{\ln \nu_{s} \Gamma(\mu+1)}{\xi_{s}}\right)^{\frac{1}{\mu}}, \bar{r}\right\}=0.7556$. The state trajectories of two subsystems and delayed FSS (39) are described as Figs 1 and 2, the switching signal is described as Fig.3.

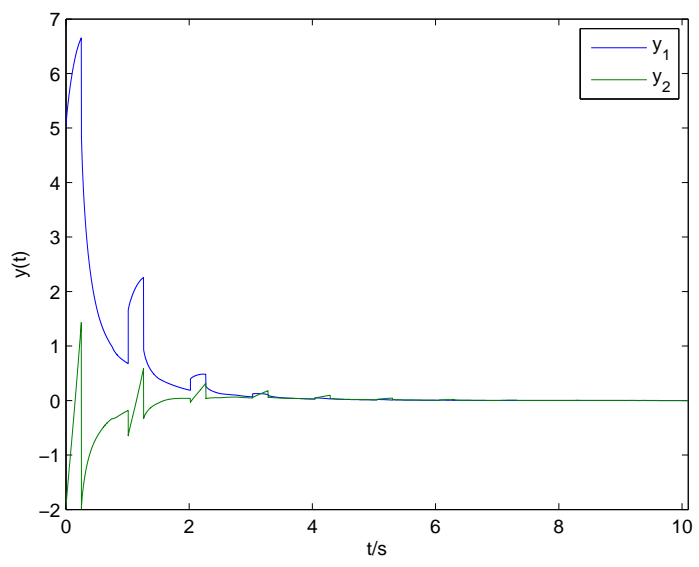

Fig.2. The state trajectories of FSS (39).

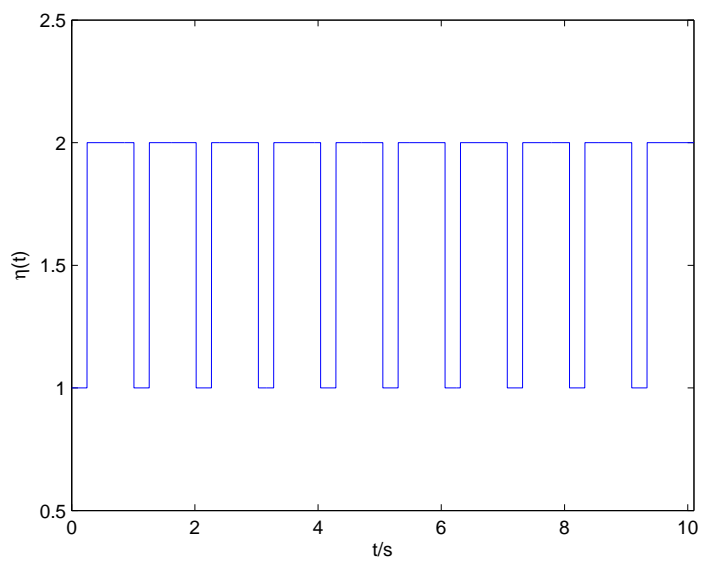

Fig.3. Switching signal of FSS (39).

Example 2 (Unstable subsystems can be consecutively activated). Consider FSS (39) equipped with four subsystems

$$
\begin{array}{ll}
A_{1}=\left(\begin{array}{cc}
1 & -0.4 \\
0.2 & 0.6
\end{array}\right), & B_{1}=\left(\begin{array}{cc}
0.1 & 0.3 \\
0.01 & 0.15
\end{array}\right), \\
A_{2}=\left(\begin{array}{cc}
-2.5 & 0.1 \\
0.2 & -3
\end{array}\right), & B_{2}=\left(\begin{array}{cc}
-0.1 & 0.2 \\
0.5 & 0.3
\end{array}\right),
\end{array}
$$




$$
\begin{array}{rlr}
A_{3}=\left(\begin{array}{cc}
0.6 & 0.1 \\
-0.5 & 0.8
\end{array}\right), & B_{3}=\left(\begin{array}{cc}
0.2 & 0.15 \\
0.1 & 0.25
\end{array}\right), \\
A_{4}=\left(\begin{array}{cc}
-3.5 & 0.5 \\
0.3 & -3
\end{array}\right), & B_{4}=\left(\begin{array}{cc}
0.3 & 0.01 \\
0.2 & 0.1
\end{array}\right),
\end{array}
$$

and $\bar{r}=1, f_{1}\left(t, y, y\left(t-r_{1}\right)\right)=\frac{1}{5} \sin y+\frac{1}{10} \tanh y\left(t-r_{1}\right), f_{2}\left(t, y, y\left(t-r_{2}\right)\right)=\frac{2}{5} \tanh y+\frac{3}{20} \sin y\left(t-r_{2}\right)$, $f_{3}\left(t, y, y\left(t-r_{3}\right)\right)=\frac{1}{10} \sin y+\frac{9}{20} \tanh y\left(t-r_{3}\right)$ and $f_{4}\left(t, y, y\left(t-r_{4}\right)\right)=\frac{1}{5} \tanh y+\frac{2}{5} \sin y\left(t-r_{4}\right)$.

Obviously, $h_{1}=0.2, l_{1}=0.1, h_{2}=0.4, l_{2}=0.15, h_{3}=0.1, l_{3}=0.45, h_{4}=0.2$ and $l_{4}=0.4$ can be chosen to meet (40). Take $P_{1}=\left(\begin{array}{cc}1.1 & 0 \\ 0 & 1.1\end{array}\right), P_{2}=\left(\begin{array}{cc}0.7 & 0 \\ 0 & 0.7\end{array}\right), P_{3}=\left(\begin{array}{ll}1 & 0 \\ 0 & 1\end{array}\right)$ and $P_{4}=\left(\begin{array}{cc}0.8 & 0 \\ 0 & 0.8\end{array}\right)$, then $\nu=1.6$ is picked to make $P_{i} \leq \nu P_{j}, i, j \in\{1,2,3,4\}$ and $i \neq j$, which means relationship (58) holds. Then we may take $\xi_{u}=4.32$ and $\xi_{s}=2.5$ to meet (56) and (57).
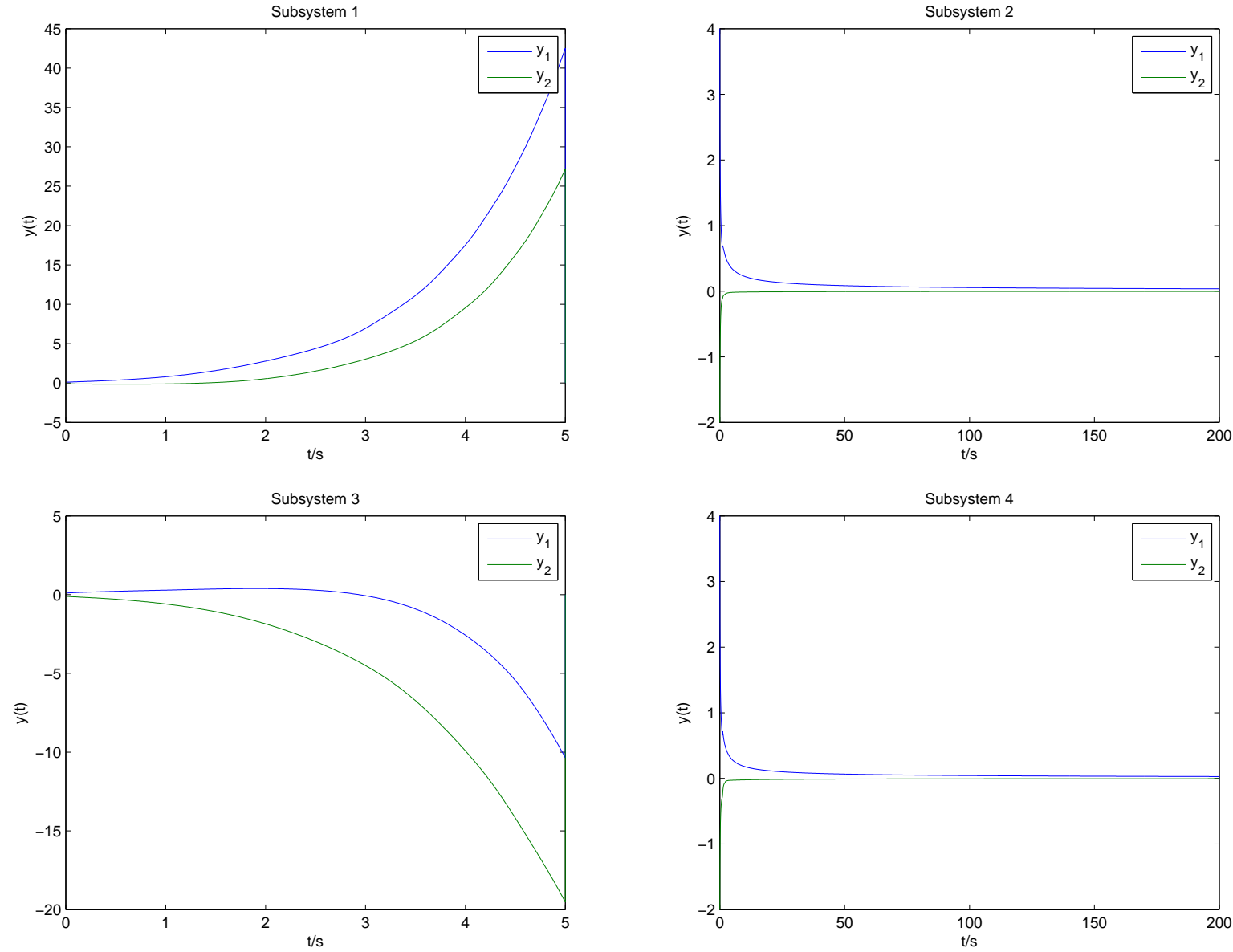

Fig.4. The state trajectories of four subsystems for delayed FSS (39).

Let $\mu=0.6, k=1.1$. Through a series of calculations, $\tau_{d s}=1.3$ and $\tau_{d u}=0.21$ can be taken 
in the light of (59). The state trajectories of four subsystems and delayed FSS (39) are describe as Figs 4 and 5, the switching signal is described as Fig.6.

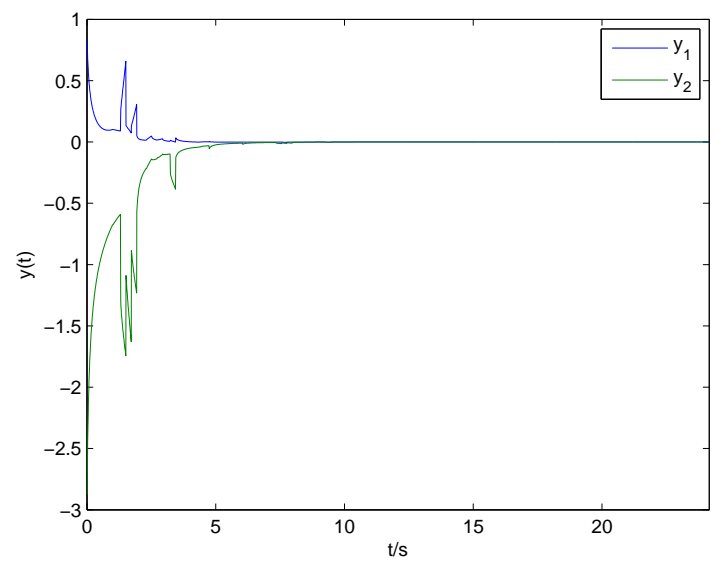

Fig.5. The state trajectories of FSS (39).

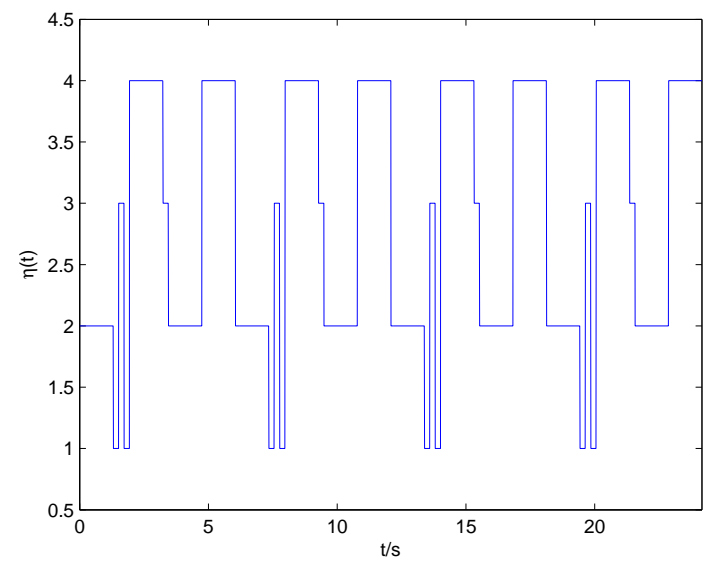

Fig.6. Switching signal of FSS (39).

\section{Conclusion}

The asymptotic stability of nonlinear FSSs with unstable and stable subsystems has been discussed in this paper. Under the assumption that unstable subsystems cannot be consecutively activated, employing fast-slow switching mechanism, MLF and dwell time technique, several algebraic conditions and the lower and upper bounds of dwell times have been derived to guarantee stability. Then, we obtain a less conservative criterion in terms of ADT technique and the property of Mittag-Leffler functions. Finally, an effective way is provided to analyse a class of delayed FSSs by virtue of fractional Razumikhin technique, the proposed method may handle the troubles well caused by delays and fractional derivatives. Our future work will focus on stability analysis of neutral and singular FSSs.

\section{Acknowledgement}

This research is funded by NNSF of China (11601003, 61803139), NSF of Anhui Province, China (2008085QA19), the Outstanding Young Talent Project of Colleges of Anhui Province, China (gxyqZD2020002). NSF of Anhui Educational Department, China (KJ2019A0004).

Availability of data and materials All data available upon request at the authors email address. 


\section{Declarations}

Conflict of interest The authors declare that they have no conflict of interest.

\section{References}

[1] B. Fahimi, A. Emadi, R.B. Sepe, A switched reluctance machine-based starter/alternator for more electric cars. IEEE Transactions on Energy Conversion 19(1) (2004) 116-124.

[2] T.J. Wall, R.T Meyer, Hybrid electric aircraft switched model optimal control. Journal of Propulsion and Power 36(4) (2020) 488-497.

[3] W. Zhang, J. Fang, W. Cui, Exponential stability of switched genetic regulatory networks with both stable and unstable subsystems. Journal of the Franklin Institute 350(8) (2013) 2322-2333.

[4] D. Liberzon, Switching in systems and control. Birkhauser, Boston, 2003.

[5] R. Ma, J. Zhao, Backstepping design for global stabilization of switched nonlinear systems in lower triangular form under arbitrary switchings. Automatica 46(11) (2010) 1819-1823.

[6] J. Zhang, Z. Han, F. Zhu, J. Huang, Stability and stabilization of positive switched systems with mode-dependent average dwell time. Nonlinear Analysis-Hybrid Systems 9 (2013) 42-55.

[7] X. Zhao, L. Zhang, P. Shi, M. Liu, Stability of switched positive linear systems with average dwell time switching. Automatica 48(6) (2012) 1132-1137.

[8] P. Yan, H. Ozbay, Stability analysis of switched time delay systems. Siam Journal on Control and Optimization 47(2) (2008) 936-949.

[9] D. Xie, H. Zhang, H. Zhang, B. Wang, Exponential stability of switched systems with unstable subsystems: a mode-dependent average dwell time approach. Circuits Systems and Signal Processing 32(6) (2013) 3093-3105.

[10] J. Wei, H. Zhi, K. Liu, X. Mu, Stability of mode-dependent linear switched singular systems with stable and unstable subsystems. Journal of the Franklin Institute 356(5) (2019) 3102-3114.

[11] L. Hou, G. Zong, Stability of switched time-delay systems via mode-dependent average dwell time switching. IEEE Access 7 (2019) 1174-1181.

[12] Z. Wang, J. Sun, G. Chen, J. Chen, Stability analysis of switched nonlinear delay systems with sampleddata inputs. International Journal of Robust and Nonlinear Control 29(14) (2019) 4700-4715.

[13] Y. Cao, Y. Li, W. Ren, Y.Q. Chen, Distributed coordination of networked fractional-order systems. IEEE Transactions on Systems Man and Cybernetics B-Cybernetics 40(2) (2010) 362-370.

[14] W. Yu, Y. Li, G. Wen, X. Yu, J. Cao, Observer design for tracking consensus in second-order multiagent systems: fractional order less than two. IEEE Transactions on Automatic Control 62(2) (2017) 894-900.

[15] S. H. HosseinNia, I. Tejado, B.M. Vinagre, Stability of fractional order switching systems. Computers and Mathematics with Applications 66(5) (2013) 585-596. 
[16] X. Zhang, Z. Wang, Stability and robust stabilization of uncertain switched fractional order systems. ISA Transactions 103 (2020) 1-9.

[17] H. Yang, B. Jiang, Stability of fractional-order switched non-linear systems. IET Control Theory and Applications 10(8) (2016) 965-970.

[18] M.V. Thuan, D. Huong, Robust finite-time stability and stabilization of a class of fractional-order switched nonlinear systems. Journal of Systems Science and Complexity 32(6) (2019) 1479-1497.

[19] J. Liang, B. Wu, L. Liu, Y.E. Wang, C. Li, Finite-time stability and finite-time boundedness of fractional order switched systems. Transactions of the Institute of Measurement and Control 41(12) (2019) 33643371.

[20] J. Zhang, X. Zhao, Y. Chen, Finite-time stability and stabilization of fractional order positive switched systems. Circuits Systems and Signal Processing 35(7) (2016) 2450-2470.

[21] T. Feng, B. Wu, L. Liu, Y.E. Wang, Finite-time stability and stabilization of fractional-order switched singular continuous-time systems. Circuits Systems and Signal Processing 38(12) (2019) 5528-5548.

[22] L. Liu, X. Cao, Z. Fu, S. Song, H. Xing, Finite-time control of uncertain fractional-order positive impulsive switched systems with mode-dependent average dwell time. Circuits Systems and Signal Processing 37(9) (2018) 3739-3755.

[23] L. Liu, X. Cao, Z. Fu, S. Song, H. Xing, Guaranteed cost finite-time control of fractional-order nonlinear positive switched systems with D-perturbations via MDADT. Journal of Systems Science and Complexity 32(3) (2019) 857-874.

[24] T. Feng, L. Guo, B. Wu, Y.Q. Chen, Stability analysis of switched fractional-order continuous-time systems. Nonlinear Dynamics 102(4) (2020) 2467-2478.

[25] G. Chen, Y. Yang, Stability of a class of nonlinear fractional order impulsive switched systems. Transactions of the Institute of Measurement and Control 39(5) (2017) 781-790.

[26] T. Zhan, S. Ma, W. Li, W. Pedrycz, Exponential stability of fractional-order switched system$\mathrm{s}$ with mode-dependent impulses and its application. IEEE transactions on cybernetics (2021) DOI:10.1109/TCYB.2021.3084977.

[27] T. Nguyen T, V. Ngoc Phat, Switching law design for finite-time stability of singular fractional-order systems with delay. IET Control Theory and Applications 13(9) (2019) 1367-1373.

[28] R. Sakthivel, S. Mohanapriya, C. K. Ahn, H.R. Karimi, Output tracking control for fractional-order positive switched systems with input time delay. IEEE Transactions on Circuits and Systems II: Express Briefs 66(6) (2019) 1013-1017.

[29] D. He, L. Xu, Exponential stability of impulsive fractional switched systems with time delays. IEEE Transactions on Circuits and Systems II 68(6) (2021) 1972-1976.

[30] R. Sipahi, F.M. Atay, S.H. Niculescu, Stability of traffic flow behavior with distributed delays modeling the memory effects of the drivers, Siam Journal on Applyed Mathematics 68(3) (2007) 738-759.

[31] J. Sabatier, Power law type long memory behaviors modeled with distributed time delay systems. Fractal and Fractional 4(1) (2020) 1-11. 
[32] I. Podlubny, Fractional Differential Equations. Academic Press, New York, 1999.

[33] A. A. Alikhanov, A priori estimates for solutions of boundary value problems for fractional-order equations. Differential Equations 46(5) (2010) 660-666.

[34] S. Liu, X.F. Zhou, X. Li, W. Jiang, Asymptotical stability of Riemann-Liouville fractional singular systems with multiple time-varying delays. Applied Mathematics Letters 65 (2017) 32-39.

[35] H. Delavari, D. Baleanu, J. Sadati, Stability analysis of Caputo fractional-order nonlinear systems revisited. Nonlinear Dynamics 67(4) (2012) 2433-2439.

[36] W. Zhu, B. Chen, J. Yang, Consensus of fractional-order multi-agent systems with input time delay. Fractional Calculus and Applied Analysis 20(1) (2017) 52-70.

[37] A. Kilbas, H. Srivastava, J. Trujillo, Theory and Applications of Fractional Differential Equations. Elsevier, Netherlands, 2006.

[38] Y. Wen, X.F. Zhou, Z. Zhang, S. Liu, Lyapunov method for nonlinear fractional differential systems with delay. Nonlinear Dynamics 82(1-2) (2015) 1015-1025. 